\title{
The Rehabilitation of a Susac Syndrome Patient: A Case Report and Clinical Review
}

\author{
Dr. Rebecca Baczuk, Independent contractor, Arnold, USA \\ Dr. Zhen Chen, Shanghai First Rehabilitation Hospital, China \\ Dr. Jeet Bhaidasna, Physical Medicine and Rehabilitation, Hampton Bays, NY USA \\ Dr. Andre Cassell, Physical Medicine and Rehabilitation, Queens NY, USA \\ Dr. Carl Cappelletti , Physical Medicine and Rehabilitation,Largo, MD USA \\ Dr. Ning Cao, MossRehab, Elkins Park, USA
}

Dr. Rebecca Baczuk ${ }^{1}$, Dr. Zhen Chen ${ }^{1}$, Dr. Jeet Bhaidasna ${ }^{2}$, Dr. Andre Cassell², Dr. Carl Cappelletti ${ }^{2}$, Dr. Ning Cao*1

Received: 制: December 12, 2018; Published: 制: January 02, 2019

*Corresponding author: Ning Cao, MossRehab, Elkins Park, USA

\begin{abstract}
Susac syndrome is a rare condition involving the brain, retina, and cochlea vasculature causing encephalopathy, visual loss due to retinal branch occlusion, and sensorineural hearing loss. Most evidence support that this disease is an acquired autoimmune disorder. Early diagnosis and aggressive treatment are important to prevent irreversible neurological damage, hearing loss, or vision loss. The objective of this case study and clinical review is to emphasize the recognition of this underdiagnosed syndrome and its treatment. Especially, the adjunct of rehabilitation treatment to pharmacological treatment may promote functional recovery.

Abbreviation: SICRET: Small Infarcts of Cochlear Retinal and Encephalic Tissues; MS: Multiple Sclerosis; DNA: Deoxyribonucleic Acid; BRAOs: Branch Retinal Artery Occlusions; AECA: Anti-Endothelial Cell Antibody; IVIg: Intravenous Immunoglobulin; PLEX: Plasma Exchange; ADL: Activities of Daily Living
\end{abstract}

\section{Introduction}

Susac syndrome was first described in 1975 by Dr. John Susac, a Neurologist then working for the U.S. Army at Walter Reed Army Hospital, who saw two patients within 3 weeks presenting with encephalopathy in the form of paranoid psychosis and personality changes, branch retinal artery occlusions, and hearing loss. He described these findings as "microangiopathy of the brain and retina" as only the precapillary arterioles ( $<100 \mathrm{um}$ ) were affected [1]. It has also been referred to as "RED- M" (Retinopathy, Encephalopathy, Deafness associated Microangiopathy) [2], "SICRET" (Small Infarcts of Cochlear, Retinal and Encephalic Tissues) syndrome, and "Retinocochleocerebral vasculopathy" [3], but the term "Susac Syndrome" has prevailed since his review of the syndrome was published in Neurology in 1994 [4]. Susac syndrome is a rare diagnosis. It is likely misdiagnosed, and therefore underdiagnosed, for three reasons:

a) Susac syndrome mimics other diseases, such as Multiple Sclerosis (MS) [5];

b) It is a rare disease with a dearth of cases, and a consequent lack of awareness of this disease; and c) It is difficult to detect due to the varied and often incomplete ("fruste forme") presentations at disease onset.

However, it is important to diagnose Susac syndrome from the myriad of other conditions it mimics, such as Multiple Sclerosis, because proper treatment and rehabilitation can halt disease progression, hasten recovery, and prevent permanent disability. That said, we are unaware of any previous publications addressing the rehabilitation of patients suffering from Susac syndrome to date. The research focus understandably so far has been on grappling with the presentation, pathophysiology, and treatment. Not surprisingly, the rehabilitation of Susac syndrome patient is also difficult, due to the involvement of the brain, vision, and hearing, all important for independent functioning, and due to the paucity of information, particularly in regard to acute and followup functionality and rehabilitation, to draw from to help guide recovery.

\section{Case Presentation}

A 51-year-old woman with a past medical history of hypertension, diabetes mellitus type 2 , chronic renal disease, 
Hepatitis C, chronic anemia, retinal detachment OD initially presented with hallucination, personality change for 6 months. Initial MRI of brain showed revealing "T2 hyperintensity lesions in the periventricular white matter representing microinfarctshallmarks of the Susac Syndrome. She received high dose of prednisone treatment resulting in improvement of her hallucination. In November 2013, she presented to an acute care hospital with acute onset left sided numbness, weakness and headache. An MRI brain revealed extensive T2/FLAIR signal hyperintensity throughout the brainstem and cerebellum and interval progression of her periventricular white matter disease, most likely the progression of her Susac syndrome. Her autoimmune workup was negative including negative ANA, anti-double-stranded DNA, ANCA, and UPEP/SPEP. Ophthalmology found total retinal detachment under ultrasound and only light perception OD; very attenuated, sclerotic vessels diffusely; and macular edema OS.

In conclusion, there was no definite evidence of Branch Retinal Artery Occlusion -one of the hallmarks of the disease, as fluorescein angiography was not available [6]. Neurology treated her empirically for Susac syndrome with plasmapheresis (PLEX) and IV steroids. The patient's mental status significantly improved after 7 sessions of PLEX followed by IV methylprednisolone, after which she was then transferred to an acute inpatient comprehensive rehabilitation (ACIR) program on a maintenance dose of prednisone. A repeat MRI brain for interval change revealed multiple predominantly subcortical bilateral cerebral hemisphere subacute infarctions consistent with Susac's microangiopathy. Additionally, an MRI of the cervical spine was negative for ischemia and a thorough skin evaluation was performed which was negative for any lesions such as livedo racemiosa $[7,8]$, On admission to ACIR, she was at a dependent level across the board in daily activities and mobility. She complained of headache, nausea, and visual and auditory hallucinations.

Her exam was notable for having a flat affect, cognitive deficits (e.g. not oriented to situation, confusion), expressive aphasia, left visual field cut, left spastic hemiplegia, Left neglect, Right dysmetria, significant lower extremity pitting edema and a healing sacral decubitus ulcer from being bedbound. In addition to mobility and activities of daily living training, she was provided low vision therapy and visual spatial orientation training for her visual impairment, vestibular therapy for vertigo, speech and language therapy to improve communication, memory and cognitive function. She also received rehabilitation nursing to provide skin care, neurogenic bowel and bladder management and neuropsychology therapy behavioral management. Her hallucinations and anxiety were significantly improved with Valproate and Seroquel which increased her participation in therapy and tolerated at least 3 hours therapy daily. As a result of three weeks acute inpatient rehabilitation, she has improved orientation and the ability to follow verbal commands. She has increased attention to the left and safety insights. She was able to direct her care and communicate her basic needs and wants. She was discharged to next level care for further rehabilitation.

\section{Discussion}

It should be noted that this patient has many confounding factors that may have contributed to her limited recovery including her age, multiple comorbidities, severe symptom at onset and possible relapse from initial episode. Many of them are not typical of Susac syndrome patients. Susac syndrome may initially present with some or all of the Susac syndrome triad of brain, eye, and inner ear involvement. The brain is the most commonly involved, but that may due to the fact that the patient may be too neurologically impaired to communicate or notice any vision or hearing loss. A review of Susac Syndrome in 2013 by Dorr et al, reported that only $13 \%$ of patients presented with all three areas of involvement of the triad; the average time lapse between first onset of symptoms and completion of the triad was 21 weeks [6].

Migraine-like headache is the most typical presenting symptom at disease onset. Other CNS manifestations of encephalopathy include confusion, memory loss, cognitive deficits, dysarthria, and psychiatric disturbances (e.g. personality change, depression, apathy, anxiety, psychosis, agitation) [9]. Retinal vasculopathy may present as vision (e.g. blurred vision, scintillation, and diplopia) or visual field deficits. Patients may have subclinical retinal pathology and hence may be asymptomatic in the presence of significant eye involvement. Branch retinal artery occlusions (BRAOs) are the most common retinal manifestation and may be found on dilated fundoscopy and with fluorescein angiography [10]. Inner ear or vestibulocochlear involvement is thought to be due to microinfarction in the arterioles of the cochlea and semicircular canals. Symptoms include sensorineural hearing loss, tinnitus, vertigo, gait ataxia, and nausea. Hearing loss is usually bilateral and asymmetric and may be either of insidious onset or it may fluctuate [10]. Other manifestations reported in association with Susac syndrome have included dermatological exanthema, cervical spinal cord lesion, and myalgia and arthralgia [7,8,10,11].

\section{Demographic Data}

Over 300 hundred cases of Susac syndrome have been reported worldwide according to Dorr et al., with the majority of reports coming from North America and Europe. The female:male ratio remains at approximately 3:1 [6]. Dorr et al, also reported a mean age of onset of 31.4 years with $81 \%$ of reported cases occurring between the ages of 16 and 40 years; however, cases have been reported from 7-72 years $[1,4,10,11]$. That said, Susac syndrome is likely underdiagnosed and may be less rare than we think; lack of awareness thwarting a rigorous workup and forme fruste presentations may thwart a timely, correct diagnosis.

\section{Laboratory and Radiographic Findings}

Presence of pathology in patients with suspected Susac syndrome was more often documented using MRI, fluorescein angiography and audiometry testing.

Encephalopathy: The classic finding on brain MRI is "snowball" and linear "spoke" lesions in the supratentorial white matter of the central corpus callosum. These represent microinfarcts which 
typically spare the periphery of the corpus collasum and which are pathognomonic for Susac Syndrome [1]. These lesions, however, differ from those typically seen in demyelinating disorders. Other lesions include those in the deep gray matter, posterior fossa, and occasionally leptomeningeal areas. EEG findings are diffusely slow. as Susac points out, "cerebral arteriography findings are almost always normal, because the involved precapillary arterioles $(<100$ um) are beyond the resolution of arteriography."

Retinopathy: On fluorescein angiography, branch retinal arterial wall "Gass syndrome" atheromatous plaques or occlusions (BRAOs) and arterial wall hyper fluorescence indicating active disease may be detected and are seen in the absence of intraocular inflammation. Fluorescein angiography helps visualize BRAO sites during an ophthalmology exam. Typically, after dye is injected the retina is visualized and the arteries appear bright white. In BRAO sites, the white vessels stop suddenly and the duration of the vessel beyond is black indicating occlusion. The visual deficits in Susac may cause substantial low visual acuity if the macula is involved, to include visual field cuts or total blindness [12,13]. Of note, BRAO is a common finding even in asymptomatic patients. One study reported BRAO on exam in $99 \%$ of patients for whom fluorescein angiography data were available [6]. Therefore, fluorescein angiography should be performed if Susac syndrome is suspected.

Hearing Loss : Audiometry reveals bilateral sensorineural hearing loss most commonly in the low and mid-range frequencies with poor speech discrimination [5]. Hearing loss may be evident on audiometry in an asymptomatic patient; therefore, audiology should also be performed as part of the workup if Susac syndrome is suspected.

Laboratory: Laboratory findings will likely not show any signs of infection. However, clotting abnormalities may be present; Dorr et al., reported coagulopathies in $22 \%$ of 181 patients for whom data were available. Lumbar puncture may reveal high CSF protein with occasional mild lymphocytic pleocytosis; the lack of oligoclonal bands and/or IgG helps discern Susac syndrome from Multiple Sclerosis [14,15].

\section{Pathophysiology}

Susac syndrome is believed to be a non-inflammatory autoimmune process. Magro, Susac et al., reported in 2011 the discovery of anti-endothelial cell antibody (AECA)-mediated microvascular injury on brain biopsy examination on indirect immunofluorescence and Western blot studies in 9 of 11 patients. Capillary C4d complement deposition resembling dermatomyositis has also been observed. AECA involvement was corroborated by Jarius et al. $[16,17]$ who analyzed CSF and serum from a patient with Susac syndrome and found complement activating IgG1 antibodies in serum but not in CSF.

\section{Clinical Course}

Susac described the clinical course of this syndrome in 2004 as "usually self-limited, fluctuating, and monophasic, lasting from 2-4 years but may range as few as 6 months or as long as 5 years [1]."
Some patients recover with little/no residual disease while others may be profoundly impaired with permanent cognitive deficits, dementia, gait dysfunction, and hearing loss; vision is usually not seriously impaired [1].

According to Rennebohm et al, 54\% of affected Susac patients were observed to have monocyclic disease (self-limiting after 2 years without recurrence) and $42 \%$ with a polycyclic form (relapses for longer than 2 years); the remaining $4 \%$ had a chronic continuous form without relapse [18]. Without immunotherapy, half of patients afflicted with Susac syndrome will likely have ongoing cognitive, visual, and auditory deficits. With treatment, however, patients usually have an excellent recovery [1].

\section{Treatment}

There are no controlled trials or series addressing treatment, and what little information is available is anecdotal. However, there is widespread agreement that Susac syndrome needs to be recognized and treated early and aggressively to prevent relapse and severe residual deficits. Since Susac syndrome appears to be an autoimmune disease, treatment is aimed at immunosuppression; however, prompt management of vision and hearing loss, debilitating psychiatric conditions, and vertigo is also important which rehabilitation treatment after acute phase would be very important.

\section{Immunotherapy}

High dose steroid therapy (both IV and oral) is the first-line treatment followed by a slow taper. In addition, antithrombotic and anticoagulation measures, antiplatelet agents and antivasospastic should be considered [19]. For those who relapse or deteriorate, intravenous immunoglobulin (IVIg), cyclophosphamide, mycophenolate mofetil, azathioprine, rituximab, and plasma exchange (PLEX) may be considered. Infliximab, a TNF inhibitor, has also been shown to be beneficial [20]. Mateen et al., reported an improvement or stabilization in eight of 11 cases treated with IVIg in the acute period (three experienced at least partial deterioration) and 8 of 9 receiving PLEX treatment [21]. To decrease or prevent cognitive deficits, blindness, and deafness, immunotherapy should be "prompt, aggressive, and sustained." Monthly administration of both IVIg and cyclophosphamide are recommended for at least six months and may be necessary for at least two years from remission $[21,22]$. Aspirin is also recommended for anticoagulation [23].

\section{Rehabilitation}

There are many challenges to rehabilitation in the patient afflicted with Susac syndrome as the brain, eye, and ear are each important contributor to independent functioning; having deficits in all three at once is devastating and requires a multidisciplinary team approach to rehabilitation. An inpatient comprehensive neurorehabilitation program including physiatrist, neuropsychology, physical therapy, occupational therapy and speech and language therapy for activities of daily living (ADLs), visuospatial orientation, transfers, mobility and ambulation, cognition, communication, and memory deficits. Visual disturbance 
due to branch retinal artery occlusion (BRAO): Immunotherapy is the mainstay of treatment to prevent retinal infarct, which would result in permanent visual deficit. Hyperbaric oxygen may improve acute onset visual symptoms in Susac's syndrome [25]. Neovascularization resulting from retinal ischemia has been treated with laser photocoagulation with some success [8].

Visual rehabilitation focused on low visual acuity and visual field cuts and can be utilized for patients with visual deficits from Susac Syndrome. Visual rehabilitation focuses on 3 main principles: compensation, substitution, and restitution. Compensation utilizes the intact vision to "compensate" for the damaged vision. Substitution focuses on corrective instruments and environmental adaptations. Restitution refers to restoring lost visual function. Prescribing for low vision rehabilitation is an integral part of any rehabilitation plan. Recent research has led to better understanding of visual functions and made possible the advent of new devices and strategies for rehabilitation of visual functions. Vision rehabilitation therapy for improvement of residual skills includes an interdisciplinary approach to maximize and integrate all of the aforementioned principles and techniques: an optometrist, ophthalmologist, or physiatrist can classically guide the rehabilitative plan for vision.

Team members include educators, occupational therapists and social workers, among others. Occupational therapists have the expertise to integrate patient functionality and vision in a home environment. Social workers can assist with any financial barriers to the rehabilitation plan [26-28]. Sensorineural hearing loss associated with Susac Syndrome was only seen in $22 \%$ of patients on initial presentation, but bilateral hearing loss will usually develop eventually. Previous study has suggested a cochlear cause, based on poor word recognition scores, absent otoacoustic emissions and normal acoustic reflexes and supported by microangiopathy indicating damage to end-arterioles supplying the apical cochlea. The treatment of the auditory manifestations of Susac Syndrome are not as clear cut, however, and much research needs to be done. Intra-tympanic injection of dexamethasone in the acute phase of the sensorineural hearing loss and tinnitus may provide transient benefit and may help justify more aggressive immunotherapy on the grounds of potential for reversibility.

In those patients with profound sensorineural hearing loss even after providing hearing aids, cochlear implants may be indicated $[29,30]$. In one study, the use of a cochlear implant resulted in $92 \%$ monosyllable and $68 \%$ medial consonant recognition in quiet conditions after 6 months of implant use. The patient in this study was also able to use the telephone after three months [29]. Current recommendation is that surgery is indicated in patients with thresholds severe enough to merit a cochlear implant. Vestibular symptoms with Susac syndrome patients such as vertigo, ataxia, and gait impairment could be caused by central or peripheral involvement [31]. A prominent jerky nystagmus could be present and is probably related to microinfarction of the membranous labyrinth [4]. Caloric tests demonstrate vestibular dysfunction that in some patients could involve the side contralateral to the side with significant cochlear [31]. Hence, vestibular rehabilitation of the patient is a crucial aspect of the rehabilitation plan. Vestibular rehabilitation is based on improving the natural phenomenon called vestibular compensation that occurs after acute vestibular disturbance or chronic and gradual misbalance Central compensation implies three main mechanisms namely adaptation, substitution and habituation. The compensation, aided by the rehabilitation aimed to compensate and/or to correct the underused or misuse of the visual, proprioceptive and vestibular inputs involved in the postural control. Encephalopathy which is laden with psychiatric features, confusion, memory loss and other cognitive changes should initially be addressed with cognitive and behavioral therapy [33]. If the patient's symptoms deteriorate in spite of psychologist intervention, they may significantly impede therapy and be detrimental to the overall wellbeing of the patient, particularly if there are psychotic features. A psychiatry consults to provide further recommendations in therapy and pharmaceutical management would be indicated. As in all other aspects of this disease, future research focusing on the rehabilitation of Susac syndrome patients is also needed. Appropriate rehabilitation will likely decrease recovery time and improve functional outcome and overall morbidity.

Because this disease is so rare, it is even more important that future research on the international level be standardized in methodology and outcomes to allow for better comparisons across studies so that our combined research is more effective. In addition to the difficulty in determining proper treatment for Susac syndrome, determining long-term management is also difficult. The polymorphisms of the disease and idiosyncratic response of patients, likely in part due to the individual differences in immune response, make planning of follow-up treatment difficult. Close multidisciplinary follow-up long-term is warranted, to include Neurology, PM\&R, PT, OT, SLP, Neuropsychology, ENT, Ophthalmology, and Rheumatology. More research into short- and long-term outcome, using standard, available measurement tools such as a Functional Independence Measure, is needed to help clinicians better understand the course of Susac syndrome, predict prognoses, and gauge response to treatment in order to provide better care.

\section{Conclusion}

Susac syndrome is a rare diagnosis which is likely underdiagnosed. It is important to have a high suspicion for, and to perform a rigorous workup of, Susac syndrome in order to distinguish it from other conditions in order to provide prompt, appropriate treatment. Accordingly, there are many challenges to rehabilitation requiring a multidisciplinary approach in Susac syndrome patients. With time, greater awareness, and continued cooperation and effort on an international level, a better understanding of this disease will unfold resulting in better care in patients afflicted with this most difficult disease.

\section{References}

1. Susac JO (2004) Susac's Syndrome. American Journal of Neuroradiology. AJNR 25(3): 351-352. 
2. Nicolle MW, Mclachlan RS (1991) Microangiopathy with retinopathy, encephalopathy, and deafness (RED-M) and systemic features. Semin Arthritis Rheum 21(3): 123-128.

3. Petty GW, Engel AG, Younge BR, Duffy J, Yanagihara T, et al. (1998) Retinocochleocerebral vasculopathy. Medicine (Baltimore) 77(1): 1240.

4. Susac JO (1994) Susac's syndrome: The triad of microangiopathy of the brain and retina with hearing loss in young women. Neurology 44(4): 591-593.

5. Crawley BK, Close A, Canto C, Harris JP (2009) Susac's Syndrome: Intratympanic therapy for hearing loss and a review of the literature. Laryngoscope 119(1): 141-144.

6. Dörr J, Krautwald S, Wildemann B, Jarius S, Ringelstein M, et al. (2013) Characteristics of Susac's Syndrome: A Review of All Reported Cases Nat Rev Neurol 9(6): 307-316.

7. Engeholm M, Leo Kottler B, Rempp H, Tobias Lindig, Holger Lerche, et al. (2013) Encephalopathic Susac's Syndrome associated with livedo racemosa in a young woman before the completion of family planning. BMC Neurol 13: 185.

8. Susac JO, Murtagh FR, Egan RA, Berger JR, Bakshi R, et al. (2003) MRI findings in Susac's syndrome. Neurology 61: 1783-1787.

9. García Carrasco M, Jiménez Hernández C, Jiménez Hernández M, Voorduin Ramos S, Mendoza Pinto C, et al. (2011) Susac's Syndrome: An Update. Autoimmunity Reviews 10(9): 548-552.

10. Susac JO, Egan RA, Rennebohm RM, Lubow M (2007) Susac's syndrome: 1975-2005 microangiopathy/autoimmune endotheliopathy. J Neuro Sci 257(1-2): 270-272.

11. Le HH, Stacy L D, Darin O (2014) A case of Susac syndrome with cervical spinal cord involvement on MRI. J Neuro Sci 337(1-2): 228-231.

12. O’Halloran HS, Pearson PA, Lee WB, Susac JO, Berger JR (1998) Microangiopathy of the brain, retina, and cochlea (Susac syndrome). A report of five cases and a review of the literature. Ophthalmology. 1998 Jun;105(6): 1038-1044.

13. Apóstolos Pereira SL, Kara José LB, Marchiori PE, Monteiro ML (2013) Unilateral central retinal artery occlusion as the sole presenting sign of Susac syndrome in a young man: case report. Arq Bras Oftalmol 76(3): 192-194.

14. Rennebohm RM, Lubow M, Jerome Rusin, Lisa Martin, Deborah M Grzybowski, et al. (2008) Aggressive immunosuppressive treatment of Susac's syndrome in an adolescent: using treatment of dermatomyositis as a model. Pediatr Rheumatol Online J 6: 3.

15. Ilka K, Jan D, Thomas D, Young P, Ringelstein EB, et al. (2014) Susac Syndrome Treated with subcutaneous immunoglobulin. Eur Neurol 71(1-2): 89-92.

16. Magro CM, Poe JC, Lubow M, Susac JO (2011) Susac syndrome: an organspecific autoimmune endotheliopathy syndrome associated with antiendothelial cell antibodies. Am J Clin Pathol 136(6): 903-912.
17. Jarius S, Neumayer B, Wandinger KP, Hartmann M, Wildemann B (2009) Anti-endothelial serum antibodies in a patient with Susac Syndrome. Journal of the Neurological Sciences 285(1-2): 259-261.

18. Rennebohm R, Susac JO, Egan RA, Daroff RB (2010) Susac's Syndromeupdate. J Neurol Sci 299(1-2): 86-91.

19. Wildemann B, Schulin C, Storch Hagenlocher B, Hacke W, Dithmar S, et al. (1996) Susac Syndrome: improvement with combined antiplatelet and calcium antagonist therapy. Stroke 27(1): 149-151.

20. Greco A, De Virgilio A, Gallo A, Fusconi M, Turchetta R, et al. (2014) Susac syndrome-Pathogenesis, clinical variants and treatment approaches. Autoimmunity Reviews 13(8): 814-821.

21. Mateen FJ, Zubkov AY, Muralidharan R, Fugate JE, Rodriguez FJ, et al. (2012) Susac Syndrome: Clinical characteristics and treatment in 29 new cases. European Journal of Neurology 2012 19(6): 800-811.

22. Ivana Vodopivec, Sashank Prasad (2016) Treatment of Susac Syndrome. Curr Treat Options Neurol 18(1): 3.

23. Kleffner I, Duning T, Lohmann H, Deppe T, Basel J, et al. (2012) A Brief Review of Susac Syndrome. Journal of the Neurological Sciences 322(12): $35-40$

24. Hardy TA, Garsia RJ, Halmagyi GM, Lewis SJ, Harrisberg B, et al. (2011) Tumor necrosis factor (TNF) inhibitor therapy in Susac's syndrome. Journal of the neurological sciences 302(1-2): 126-128.

25. Li HK, Dejean BJ, Tang RA (1996) Reversal of visual loss with hyperbaric oxygen treatment in a patient with Susac syndrome. Ophthalmology 103(12): 2091-2098.

26. Markowitz SN (2006) Principles of modern low vision rehabilitation. Canadian Journal of Ophthalmology 41(3): 289-312.

27. Khan S, Leung E, Jay WM (2008) Stroke and visual rehabilitation. Top Stroke Rehabil 15(1): 27-36.

28. Henriques DY, Cressman EK (2012) Visuomotor Adaptation and Proprioceptive Recalibration. Journal of Motor Behavior 44(6): 435-444.

29. Bittencourt AG, Santos AF, Goffi Gomez MV, Kutscher K, Tsuji RK, et al. (2011) Microangiopathy of the inner ear, deafness, and cochlear implantation in a patient with Susac syndrome. Acta Otolaryngol 131(10): 1123-1128.

30. Grover N, Whiteside OJ, Ramsden JD (2011) Susac Syndrome: outcome of unilateral cochlear implantation. J Laryngol Otol 125(8): 856-858.

31. Han BI, Song HS, Kim JS (2011) Vestibular Rehabilitation therapy: Review of indications. Mechanisms, and Key exercise, J Clin Neurol 7(4): 184-196.

32. Fox RJ, Costello F, Judkins AR, Galetta SL, Maguire AM, et al. (2006) Treatment of Susac syndrome with gamma globulin and corticosteroids. J Neruo Sci 251(1-2): 17-22.

33. Aubart Cohen F, Klein I, Alexandra JF, Bodaghi B, Doan S, et al. (2007) Long term outcome in Susac syndrome. Medicine 86(2): 93-102. 


\section{ISSN: 2574-1241}

DOI: 10.26717/BJSTR.2019.12.002287

Ning Cao. Biomed J Sci \& Tech Res

This work is licensed under Creative

Commons Attribution 4.0 License

Submission Link: https://biomedres.us/submit-manuscript.php

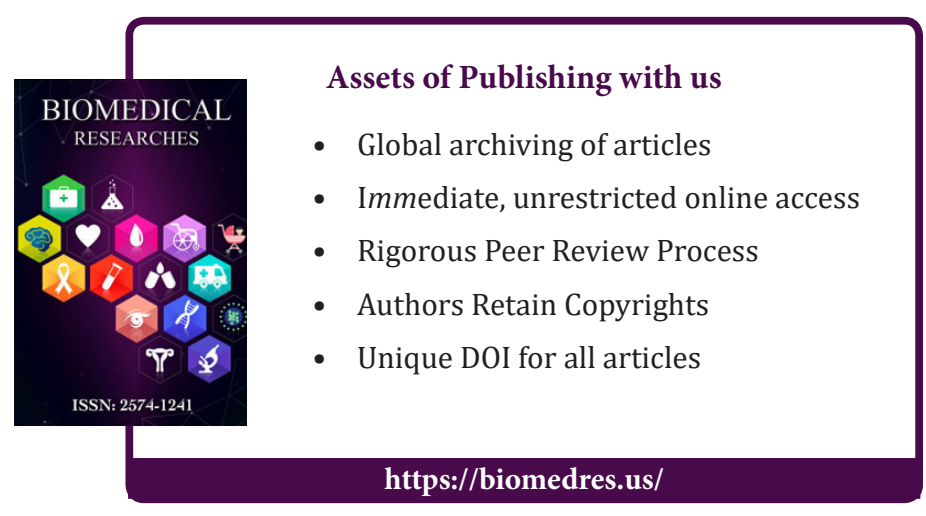

